\title{
Cell Therapies for ARDS: The Importance of Proper Study Design-Letter to the Editor-in-Chief and his Response
}

\section{Daniel J Weiss ${ }^{1 *}$ and Katarina Le Blanc ${ }^{2 *}$}

${ }^{1}$ University of Vermont College of Medicine, USA

${ }^{2}$ Karolinska Institutet, Sweden

Marek Malecki MD, PhD

Editor-in-Chief

Journal of Stem Cell Research and Therapy

Dear Dr. Malecki,

We read with interest the recently published manuscript, "Successful Reversal of Acute Lung Injury using Placenta-Derived Decidual Cells", (J Stem Cell Res Ther 4: 244.doi.org/10.4172/2157-7633.1000244). However, we are puzzled by a number of things in this manuscript. A number of significant concerns are detailed below.

1. The major concern is the implication of causality. In both the title and at several points in the manuscript, for example the first sentence of the discussion, the author's claims or imply that the clinical improvement observed was a direct result of actions of the stromal cells administered. However, clinical improvement coincided not only with stromal cell infusion but also with neutrophil engraftment in a previously neutropenic patient and occurred 3 days after initiation of antibiotics as well as administration of corticosteroid and changes in overall fluid status in the setting of diuretic administration. As such, it is impossible to directly ascribe the clinical and inflammatory improvements to the stromal cell administration. All the more so as, further detailed below, the clinical information presented is incomplete and in some respects confusing and inconsistent.

Moreover it is inaccurate and arguably irresponsible to ascribe a clinical benefit to a new potential therapeutic approach based on a single anecdotal case report. This can only be done in the setting of a well-designed and well conducted randomized double-blinded clinical trial. An example of this is the one currently being conducted at UCSF, of which the results of the phase 1 arm were just published in Lancet Respiratory Medicine (Wilson et al. Mesenchymal stem (stromal) cells for treatment of ARDS: a phase 1 clinical trial. Lancet Respiratory Medicine 3:24-32, 2015). An accompanying editorial highlights the importance of appropriate clinical trials in ascribing any potential therapeutic benefit of MSC administration in patients with ARDS (Weiss DJ. Lancet Respiratory Medicine 3:2-3, 2015).

2. A second major concern is that the authors have apparently administered the decidual stromal cells (DSCs) to 55 patients yet no outcomes or results of any of these patients have been presented. Why then do the authors present a single anecdotal experience in the current case report, particularly one in which there is no clear or compelling evidence that DSC administration had any direct efficacy?

3. A third major concern is the timing and concept of compassionate use DSC administration in the course of this patient's clinical illness. The patient was still on face mask oxygen and although "exhausted" was not on mechanical ventilation and was in the midst of a number of ongoing therapeutic interventions mentioned above and further discussed below. As such, there were still number of supportive and clinical intervention options in progress and also still available. As such, it is hard to grasp how a compassionate use decision, i.e. failure of all other available treatment/supportive options, was reached.

4. A fourth major concern is the incomplete presentation and

description of the clinical parameters. A fuller understanding of these would suggest other potential mechanisms for the patient's improvement. These are listed in detail as follows:

a. The statement is made in the abstract and repeated elsewhere that oxygen saturation instantly or within minutes improved following DSC administration. However, in Figure 2A, the time scale (X-axis) is spread over days and it is not clear from the data presented that the saturations "instantly" improved. Can the authors better define "instantly" and provide the accompanying data. It is also important to note that in the COPD trial discussed and also in the now published phase 1 trial of MSCs for ARDS, there was no change in oxygen saturation during or shortly after MSC infusions. This is felt to be an important indicator of safety and argued against clinically significant pulmonary emboli resulting from the cell administration. Can authors better explain their thinking as to why oxygen saturations increased after DSC infusion?

b. This patient appears to have had pulmonary edema. This is evidenced by a number of pieces of clinical data including chest $\mathrm{X}$-rays on $\mathrm{d}+10$ and $\mathrm{d}+11$ (Figures $3 \mathrm{~A}$ and $3 \mathrm{~B}$ ) that appears to show textbook examples of "batwing" pulmonary edema. In parallel, an increase in weight, an increase in serum creatinine suggesting acute kidney injury, increased central venous pressure, significant fluid volume loading with transfusions of erythrocytes and platelets, and presumably decreased plasma oncotic pressure in the setting of decreased serum albumin all suggest that the patient did have at least some component of hydrostatic pulmonary edema. The patient had also received Cytoxan, which may have contributed to a cardiomyopathy and subsequent heart failure.

The relevant clinical data presented is confusing:

1) "Blood pressure was $120 / 60 \mathrm{mmHg}$. What relevance does this single blood pressure measurement on day 9 have? What are necessary to understand are trends in blood pressure over time during this period.

2) "Due to sepsis, the hemoglobin decreased from 100 to $60 \mathrm{~g} / \mathrm{L}$ ". This is confusing. Presumably the patient was septic in the setting of bacteremia, fever, and neutropenia. However, had he developed diffuse intravascular coagulation? As with blood pressure, serial measures of

*Corresponding authors: Daniel J Weiss, Professor of Medicine, University of Vermont College of Medicine, Burlington, Vermont 05405, USA, Tel: 802-656-3131; E-mail: daniel.weiss@med.uvm.edu

Katarina Le Blanc, Professor in Clinical Stem Cell Research, Karolinska Institutet, Stockholm, Sweden, E-mail: Katarina.Leblanc@ki.se

Received February 22, 2015; Accepted March 11, 2015; Published March 13 2015

Citation: Weiss DJ, Blanc KL (2015) Cell Therapies for ARDS: The Importance of Proper Study Design-Letter to the Editor-in-Chief and his Response. J Stem Cell Res Ther 5: 268. doi:10.4172/2157-7633.1000268

Copyright: ( 2015 Weiss DJ, et al. This is an open-access article distributed unde the terms of the Creative Commons Attribution License, which permits unrestricted use, distribution, and reproduction in any medium, provided the original author and source are credited. 
hemogblobin and other hematologic parameters are necessary. Could some of the pulmonary infiltrates have reflected diffuse alveolar hemorrhage?

3) “...serum creatinine increased.... Again, did the patient have an acute kidney injury?

4) Urine output is listed at 4.9 liters on day 10 and 4.8 liters on day 11. However, total input is not listed. It is critical to understand if the patient was in an overall positive or negative fluid balance on each of these days.

5) "Central venous pressures ranged from 1.5-8 mm Hg". No context is given for this statement. It is further important to understand the trends in CVP during the patient's clinical course.

c. The patient likely had pneumonia in the setting of persistent high fever, bacteremia, and neutropenia.

d. The patient may have also had transfusion-associated acute lung injury (TRALI) in the setting of erythrocyte and platelet transfusions.

e. The patient had received methotrexate which may have contributed to a subacute pneumonitis.

f. As discussed above, the patient may have had diffuse alveolar hemorrhage.

All of these are relevant potential etiologies of the patient's respiratory failure. What is critically important is that the patient had a number of concurrent different therapies during the same time period in which the DSCs were administered. These included antibiotics, diuretics, albumin, and corticosteroids, each of which could have effect on the different potential etiologies of respiratory failure and pulmonary infiltrates.

5. Moreover, the patient appears to have started engrafting coincident in time with DSC administration. The presence of functional WBCs may well have contributed to resolution of a potential pneumonia with improvement in pulmonary infiltrates, oxygenation, and circulating inflammatory mediators.

6. Circulating levels of several inflammatory cytokine levels are lowered 3 hours and then one week after DSC administration compared to "Before DSC" levels. However, "Before DSC" is not clarified and there is no sense of how the cytokine values were already trending. Arguably, they all could have been much higher than the "Before DSC" value and thus already decreasing, perhaps in response to the effects of the other ongoing therapeutic interventions. Further, no reference levels are given. Hence, causality with respect to DSC administration cannot be made in this context.

5. The paper refers to the group's previously published experience using bone marrow MSC. Of the authors on the present paper, only the first author of the present paper was involved in those studies. Notably, Dr. Ringden was also senior author of a long-term followup of MSC-treated patients (von Bahr et al, 2012, BBMT 18; 557-564) in which potential side effects were compared to a control group of acute GvHD (including steroid-responsive patients) without MSC treatment. When adjusting for survival, in contrast to what is stated in the current manuscript, there was no increased incidence of invasive fungal infections in MSC-treated patients as compared to non-MSCtreated (unpublished data). Although the von Bahr manuscript was published without a control group, as the selection of MSC-treatment was not randomized introducing a bias in the material and the groups were thus not comparable, Dr. Ringden is aware of these results. It is thus hard to understand how the statement on increased risk of fungal infections was made in the current manuscript. The referenced publication (Remberger, Ringdén, 2012, Int J Hematol) published on the same patients should be questioned on this basis.

6. The authors state that MSCs home to damaged tissues but no such evidence exists in humans. Further, the MSCs don't home to lung either, rather currently available data suggests that, following systemic administration, MSCs will transiently lodge in the pulmonary capillary bed for 24-48 hours prior to being cleared by still as yet poorly understood mechanisms. Some pre-clinical data suggests that the residence or survival of MSCs in the lung capillary beds may be longer in the setting of acute lung injury. However, there is little correlative clinical data yet available. The reference on homing quoted in the current manuscript refers to an anecdotal finding of MSC-donor DNA detected in damaged tissue. Yet, when analysed in a larger cohort of patients, no correlation was found between detection of MSC-donor DNA and sites of tissue injury (von Bahr et al, Stem Cells 2012). As Dr. Ringden is a co-author of this larger analysis, this should be a known fact when writing the present paper.

As such, providing a clinical suggestion based on a misquoting of available literature and on another anecdotal experience in which no clear association was shown between MSC administration and development of invasive pulmonary aspergillosis, an unfortunately not uncommon occurrence in allogeneic bone marrow transplant recipients, is again unwarranted and arguably irresponsible.

7. The authors state that due to a patent held by Osiris, it is difficult to conduct clinical academic trials in USA. This statement is simply incorrect. Firstly, the patent previously held by Osiris, has been sold to other companies. Secondly, no legal difficulties prevent conduct of academic US based clinical trials. Indeed the National Institutes of Health has a program, the Production Assistance of Cell Therapy (PACT) program that is specifically designed to provide bone marrowderived MSCs and other cells for academic-based clinical investigations. This has borne recent fruition in the above-mentioned Phase 1 trials of bone marrow-derived cells in patients with ARDS (Wilson et al Lancet Respiratory Medicine 3:24-32, 2015). Further, there are a number of other companies conducting industry-based clinical trials of MSCs derived from bone marrow, adipose tissue, placenta, and other sources for a range of diseases. A simple search of the clinicaltrials.gov website would have revealed this information.

There are also a number of smaller but still important inaccuracies and misquotations in the article.

8. While the authors are correct in quoting the American-European Consensus Conference on ARDS for defining ALI or ARDS, these criteria are over 20 years old and were updated 3 years ago (Ranieri VM, Rubenfeld GD, Thompson BT et al. Acute respiratory distress syndrome: the Berlin Definition. Jama 2012; 307: 2526-2533.). The authors should utilize and reference these updated criteria.

9. In the first sentence of the second introductory paragraph, the statement "...MSCs have been widely used..." is perhaps better thought of as "...MSCs have been widely investigated...”. To date, despite much investigation and many anecdotal reports and small case series, appropriate larger scale clinical trials have demonstrated only few clear successful clinical approaches using MSCs for their immunomodulatory actions.

10. The statement is made in both the abstract and the introduction that the fetus is protected from the mother's immune system by the placenta and the fetal membranes. This is not a fully accurate statement. As immune cells from the mother can enter into the fetus, the decidua may limit cellular traffic but that does not prevent potentially harmful effects of the maternal immune system. 
11. "We switched to DSCs for clinical use because they have a more reproducible immunomodulatory effect than MSCs, they are easier to obtain, and they have better expansion capacity than MSCs [11,12]."

It is unclear how the authors can make this statement since the given references contain no comparison of either expansion potential or immune suppressive effects of MSC vs DSC, either in vitro or specifically not in vivo.

12. "Preclinical studies have suggested that MSCs may be of value in treating ALI [13-16]". Reference 13 discusses a mouse model of hyperoxia exposure while reference 16 is a mouse model of sepsis. References 14 and 15 are more relevant but older. Another reference is quoted in which amnion epithelial cells administered to bleomycininjured mice can reduce acute lung injury and thus prevent development of subsequent lung fibrosis. While appreciating the attempt to provide a framework, there are a number of more recent and relevant references for potential consideration by the authors.

13. Infusing one million cells per kg body weight over a 5 minute period seems fast. What was the rationale for this rate of infusion and how does it compare with other clinical approaches. For example, in the COPD trial, MSCs were infused over a 60 minute period.

14. The authors state that the in vivo anti-inflammatory actions of the DSCs "probably" dampened potential pro-inflammatory actions of engrafting neutrophils. While a potentially attractive hypothesis, what data backs up this specific hypothesis?

15. "DSCs require cell contact to be immunosuppressive". Is this really the case? The authors' quote one reference that describes investigation of ex vivo actions of DSCs on T cells. However, as the authors are undoubtedly aware, there is a rapidly developing literature demonstrating that conditioned media and factors released by MSCs and isolated from conditioned media, including microvesicle particles, can mitigate most immunomodulatory actions of MSCs both in in vitro models and increasingly so in in vivo models of a widening spectrum of diseases. This includes experimental models of lung injuries.

Do the authors truly believe that DSCs will be completely unlike MSCs isolated from bone marrow, adipose tissue, and other sources in this respect?

16. "We used a fluid-conservative strategy, which has proven to be effective in ARDS..... If this is indeed the case, it needs to be presented and clarified in the methods/results section. Can the authors quote the appropriate New England Journal Medicine reference that has set a working standard for a fluid-conservative approach (Wiedemann HP. Wheeler AP. Bernard GR. Thompson BT. Hayden D. deBoisblanc B. Connors AF Jr. Hite RD. Harabin AL. National Heart, Lung, and Blood Institute Acute Respiratory Distress Syndrome (ARDS) Clinical Trials Network. Comparison of two fluid-management strategies in acute lung injury. New England Journal of Medicine 2006; 354(24): 2564-75).

17. "We also wanted to avoid ventilator assistance because this may worsen lung injury in patients who have undergone HSCT". What is the specific data supporting this contention other than retrospective observations of high mortality of HSCT patients on ventilator support, rather reflecting poor outcome of severe infection and other organ failures.

18. "There is some concern with the report of pulmonary embolism following infusion of adipose-derived MSCs (28)" It is unclear what the intent of the author's is with this statement. As they had discussed in preceding statements, a growing large body of literature has not shown signs of acute toxicity with MSC administration. This includes clinical signs or symptoms of clinically significant pulmonary emboli. The reference quoted is from an obscure journal and apparently is concerned with an unusual form of familial pulmonary embolism.

19. The COPD trial was underpowered for efficacy. That was clearly stated in the article describing that trial. This should be clarified in the current manuscript.

Thank you for your consideration in all of this.

Sincerely,Sincerely,

\section{Dan Weiss}

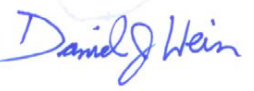

Daniel J. Weiss M.D., Ph.D.

Professor of Medicine

Pulmonary and Critical Care

Vermont Lung Center

University of Vermont College of Medicine

Burlington, Vermont 05405

\section{Katarina Le Blanc}

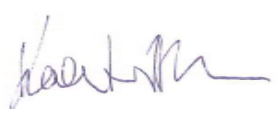

Katarina Le Blanc, M.D., Ph.D.

Professor in Clinical Stem Cell Research

Karolinska Institutet, KI

Alfred Nobels Allé 8

SE-141 83 Huddinge

Stockholm, Sweden

\section{Editor-in-Chief Response to the Letter}

\section{Greetings, Dr Weiss and Dr LeBlanc,}

I thank you for your letter. I surely support its publication. In fact, I commend you for agreeing to publish it as an Open Letter to the Authors. We asked them to respond to your questions. We will surely publish their response.

As you know, at the JSCRT, I promote not only Open Access, but also Open Pre- and Post-publishing Review. In my humble opinion, this is the way to assure full transparency of the review and publication processes, revealing potential and real conflicts of interests, assuring fair and equal access of all authors for disseminating the results of their works, as well as assuring accountability of the authors. Therefore, I especially thank you for contributing to this endeavor.
Sincerely,
Marek Malecki MD PhD
Editor-In-Chief
Marek Malecki MD PhD
President
Website: http://pbmef.org
Telephone: 415-713-4370
Fax: 415-713-4371
Email: marekmaleckimdphd@pbmef.org 